\title{
Primary standard of optical power operating at room temperature
}

\author{
Timo Dönsberg ${ }^{1,2}$, Meelis Sildoja ${ }^{2}$, Farshid Manoocheri ${ }^{1,2}$, Mikko Merimaa ${ }^{1}$, Leo Petroff ${ }^{3}$ and Erkki Ikonen ${ }^{1,2}$ \\ ${ }^{1}$ Centre for Metrology and Accreditation (MIKES), P.O. Box 9, FI-02151 Espoo, Finland \\ ${ }^{2}$ Metrology Research Institute, Aalto University, P.O. Box 13000, FI-00076 Aalto, Finland \\ ${ }^{3}$ Fitecom Oy, Peräsimentie 8, FI-03100 Nummela, Finland
}

\begin{abstract}
The Predictable Quantum Efficient Detector (PQED) is evaluated as a new primary standard of optical power. Design and characterization results are presented for a new compact room temperature PQED that consists of two custom-made induced junction photodiodes mounted in a wedged trap configuration. The detector assembly includes a window aligned in Brewster angle in front of the photodiodes for high transmission of $p$ polarized light. The detector can also be operated without the window, in which case a dry nitrogen flow system is utilized to prevent dust contamination of the photodiodes. Measurements of individual detectors at the wavelength of $488 \mathrm{~nm}$ indicate that reflectance and internal quantum efficiency are consistent within $14 \mathrm{ppm}$ and $10 \mathrm{ppm}$ (ppm = part per million), respectively, and agree with the predicted values. The measured photocurrent ratio of the two photodiodes confirms the predicted value for $s$ and $p$ polarized light, and the spatial variation in the photocurrent ratio can be used to estimate the uniformity in the thickness of the silicon dioxide layer on the surface of the photodiodes. In addition, the spatial non-uniformity of the responsivity of the PQED is an order of magnitude lower than that of single photodiodes. Such data provide evidence that the room temperature PQED may replace the cryogenic radiometer as a primary standard of optical power in the visible wavelength range.
\end{abstract}

\section{Introduction}

Cryogenic electrical substitution radiometers [1-4] are currently used as the primary standard of optical power. These sophisticated devices can typically achieve standard measurement uncertainty of around $100 \mathrm{ppm}$, or even as low as $40 \mathrm{ppm}[3,4]$, but they require operation near the temperature of $10 \mathrm{~K}$. An alternative approach is to use a silicon photodetector with well-known responsivity as the standard of optical power [5-9]. Ideally a photodetector converts each incident photon into a charge carrier, which is then collected without any losses. Therefore, the ideal spectral responsivity $e \lambda / c h$ is only dependent on fundamental constants $c, e, h$ and the vacuum wavelength $\lambda$. However, in practice, the responsivity of a photodetector is reduced by reflectance and the internal quantum deficiency (IQD), corresponding to the relative number of absorbed photons not producing a collected charge carrier. The combined effect of these two is called external quantum deficiency (EQD).

As compared to regular diffused $p-n$ junction photodiodes, the high-efficiency induced junction photodiodes [10-12] may have considerably smaller charge carrier losses. The effect of reflectance, on the other hand, can be reduced by Brewster-angle operation
[13] or by assembling the photodiodes into a lighttrapping configuration [9,14-17]. These loss reduction methods are used to construct the Predictable Quantum Efficient Detector (PQED) $[18,19]$. It consists of two induced junction photodiodes with tailored design parameters suitable for the particular light-trapping configuration.

At room temperature, the spectral responsivity of the PQED can be predicted within the standard uncertainty of $70 \mathrm{ppm}$ in the visible wavelength range [12]. Measurements against cryogenic radiometers at $476 \mathrm{~nm}$, $532 \mathrm{~nm}$ and $760 \mathrm{~nm}$ confirm the predicted responsivity [19]. The uncertainty of the predicted responsivity of the room temperature PQED is comparable with that of the cryogenic radiometer suggesting that the PQED can be used as a primary standard of optical power in the visible wavelength range.

The room temperature performance of the PQED has earlier been tested mainly with detectors assembled in liquid nitrogen cryostats. The design and characterization results of a new room temperature PQED are presented here. The developed detectors provide the benefits of compact size and ease of use similar to conventional trap detectors and demonstrate the improvement of the spatial uniformity by about a factor of ten as compared to single photodiodes. Several detectors were assembled and 
characterized. Characterization results indicate that the new device meets the expectations for a high quality primary standard.

\section{Design of the detector}

The type of induced junction photodiodes used in the new detector design are described and characterized in [18] and [19]. In these photodiodes the depletion region is produced by the surface charges in the silicon dioxide layer [10], which improves the collection efficiency of charge carriers. The collection efficiency is further increased by applying a reverse bias voltage that extends the depletion region farther into the bulk silicon. As compared to diffused junction photodiodes, the induced junction produces a high electric field in a more advantageous position close to the oxide-silicon interface, where localized intermediate states acting as recombination centers are inevitable. The electric field separates electrons and holes generated near the silicon surface before significant recombination takes place.

The wedged light-trapping configuration of the two photodiodes is illustrated in Figure. 1. One of the photodiodes has an oxide layer thickness of $300 \mathrm{~nm}$ and the other $220 \mathrm{~nm}$. Seven reflections of the incident beam take place and a fraction of around tens of ppm is then reflected back. This residual reflection can be used for alignment and diagnostics of the assembly.

The photodiodes are encased in a cylindrical body that has a maximum aperture of $10 \mathrm{~mm}$ in diameter. The air-tight assembly, shown in Figure 2, has a length of $215 \mathrm{~mm}$ and an outer diameter of $75 \mathrm{~mm}$. The $6 \mathrm{~mm}$ thick fused silica window in front of the detector is aligned in Brewster angle for high transmission of $p$ polarized light. The design is such that the plane of $p$ polarized incidence is the same for the Brewster window and photodiodes. The window holder is connected to the body of the detector using flexible bellows. This allows precise alignment of the window using fine-threaded screws. With the exception of stainless steel bellows, the body of the detector is made of black anodized aluminum.

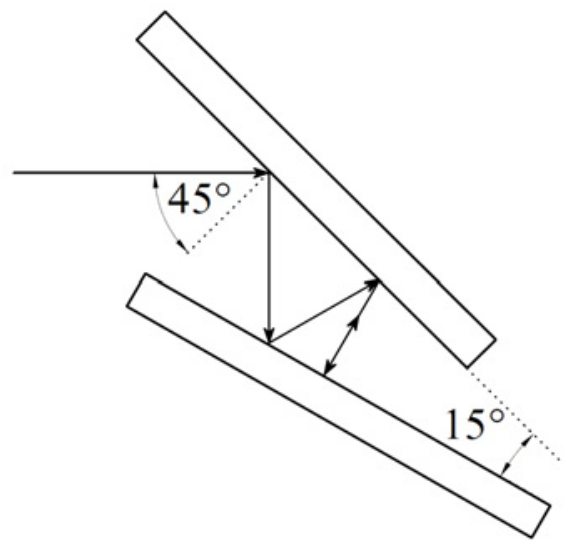

Figure 1. Schematic of the light-trapping assembly. Similar detector structures are thoroughly studied in [17].

The detector can also be used without the window, in which case the need for window transmittance correction, which is a major contribution to the uncertainty, is eliminated. The dust and moisture contamination of the photodiodes is prevented by using a dry nitrogen flow system, where the filtered dry nitrogen enters via an inlet at the back of the detector, flows around the photodiodes and exits through the aperture in front of the photodiodes. In typical operation conditions the dry nitrogen consumption is less than two liters per minute.

The photodiodes are connected using two BNC connectors at the back of the detector, allowing the photocurrents to be measured separately, when required. Total photocurrent is measured by connecting the signals from the photodiodes in parallel and using a common bias voltage and current-to-voltage converter. The BNC connectors are insulated from the detector body, enabling a floating measurement of photocurrents and a separate grounding for the housing.

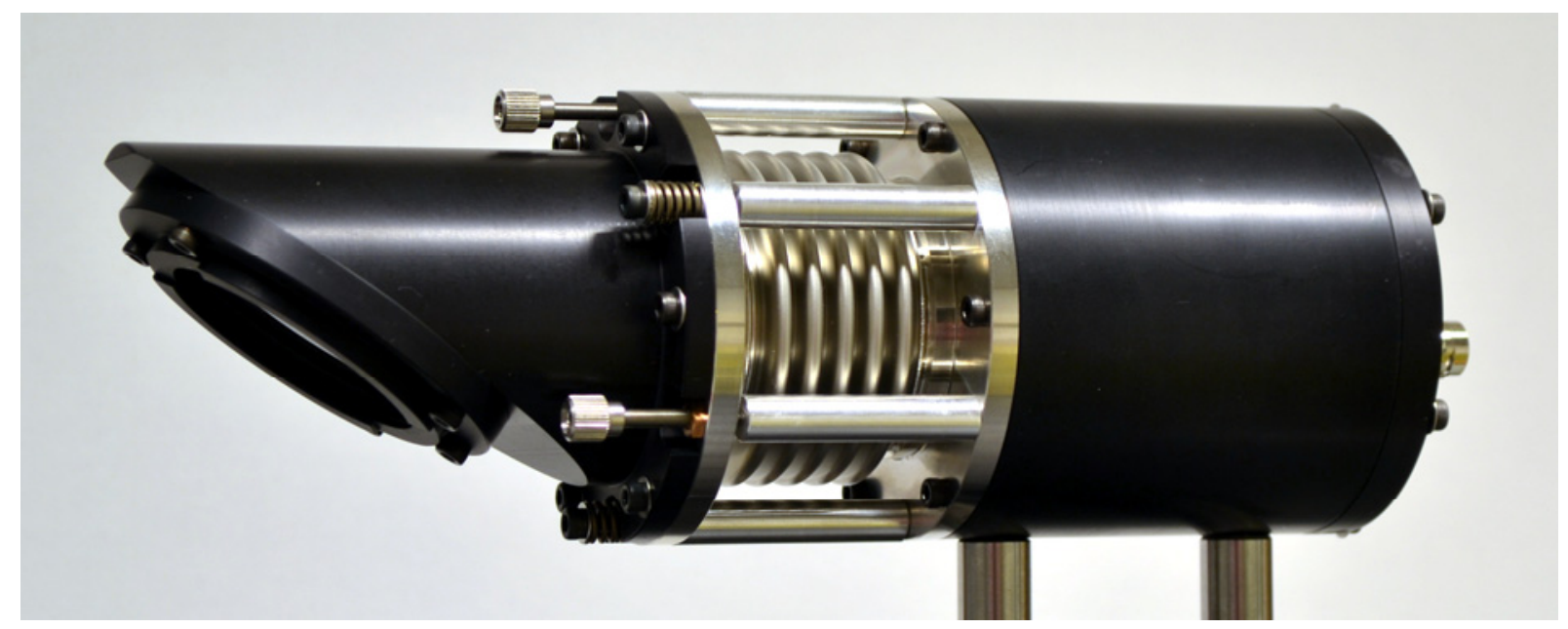

Figure 2. Photograph of the room temperature PQED showing (from left to right) the Brewster window, adjustment screws, flexible bellows and the photodiode chamber. The total length is $215 \mathrm{~mm}$ and outer diameter of the photodiode chamber $75 \mathrm{~mm}$. 


\section{Characterization measurements}

Several PQEDs were assembled and characterized for spatial uniformity of responsivity, photocurrent ratio, specular reflectance, and relative and absolute responsivity. All measurements were conducted at room temperature using an intensity stabilized argon ion laser operated at the vacuum wavelength of $488 \mathrm{~nm}$. The optical power was approximately $200 \mu \mathrm{W}$ and the incident light was $p$ polarized in all the measurements, unless otherwise noted. Photodiodes were reverse biased with 5 volts and the photocurrents were measured separately.

For all measurements, with the exception of spatial uniformity measurements, the detector was aligned such that the beam hits the central part of the active area of the detector. This was achieved by moving the PQED in front of the laser beam using an $x y$ translator and finding the edges of the active area. Central location was defined as the midpoint of the edge-to-edge distances along horizontal and vertical axes.

\subsection{Spatial uniformity of responsivity}

The spatial uniformity of the responsivity was measured with a two-axis linear translator. The active area was scanned in both directions with $0.5 \mathrm{~mm}$ steps from -4 to $+4 \mathrm{~mm}$ relative to the center of the active area. The drift in the laser power level was found to have an effect of around $40 \mathrm{ppm}$ in responsivity during such long term measurements. The effect due to instability was reduced by a factor of 10 by measuring a reference signal from the center spot before and after each movement of the PQED.

A measurement result of spatial uniformity of responsivity is presented in Figure 3. The data indicate that with a $1 / e^{2}$ laser beam diameter of $1.2 \mathrm{~mm}$, the responsivity changed only $50 \mathrm{ppm}$ within a diameter of $4 \mathrm{~mm}$. Thus, the non-uniformity of the PQED is about ten times lower than the non-uniformity of single induced junction photodiodes over similar area, as measured in [19], and can match that of best conventional silicon trap detectors [20-22].

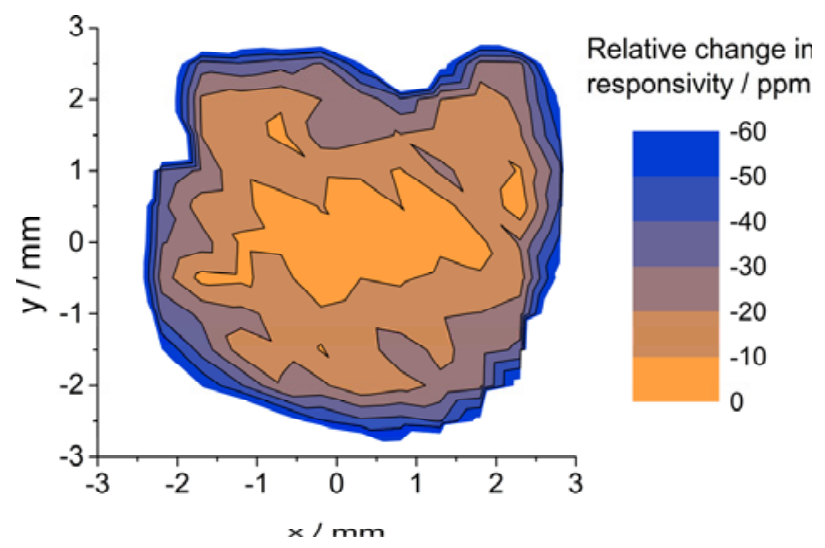

Figure 3. Spatial uniformity of responsivity measured without the Brewster window of the PQED. Values are given as a relative deviation from the measured peak value. Reduced responsivity at the edges of the measured area may be caused by loss of weak scattered radiation outside the main beam.

\subsection{Photocurrent ratio}

As the photocurrents can be measured separately, the spatial uniformity of the photocurrent ratio is easily obtained and independent of laser power drift. The photocurrent ratio of the PQED has typically a spatial variation of about $1 \%$ peak-to-peak, as seen in Figure 4. As the rather large spatial variation in the photocurrent ratio is not seen in the spatial uniformity of the responsivity, it can be concluded that the variation in the photocurrent ratio is solely due to variations in photodiode reflectance and the IQD of the photodiode is spatially uniform within approximately $50 \mathrm{ppm}$. The spatial variation of the photocurrent ratio can be explained by peak-to-peak deviations of $0.7 \mathrm{~nm}$ to $1.5 \mathrm{~nm}$ in the oxide thicknesses. This range is in agreement with the oxide thickness non-uniformities measured in [23].

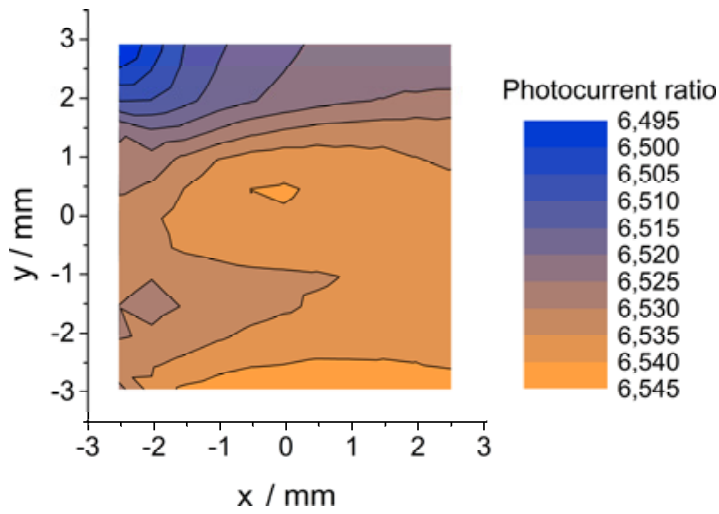

Figure 4. Spatial variation in the photocurrent ratio of the same $\mathrm{PQED}$ as in Figure 3.

The calculated and measured photocurrent ratios at the center of the detector with different combinations of $s$ and $p$ polarized components in the incident laser beam are shown in Figure 5. The nominal value and the uncertainty corridor are calculated using detector and photodiode parameter values and uncertainties obtained from [18]. The most significant contributions are caused by uncertainty of the angle between photodiodes and of the oxide thicknesses of the photodiodes. The measured data are in agreement with the prediction that with $s$ polarized light the photocurrent ratio is more sensitive to variations in the detector and photodiode parameters than with $p$ polarized light.

The photocurrent ratio depends on the angle of the incidence on the first photodiode, especially with $s$ polarized light. At the wavelength of $488 \mathrm{~nm}$, a deviation of 0.1 degree in the incidence angle has an effect of approximately $1 \%$ and $0.1 \%$ on the photocurrent ratio with $s$ and $p$ polarized light, respectively. This property can be useful for aligning a PQED with known photocurrent ratio, if the back-reflection from the detector cannot be directly observed. Furthermore, when an incoherent light source with known polarization is being used, proper alignment of the detector may be more easily obtained by adjusting the photocurrent ratio than by observing the weak reflected light. 


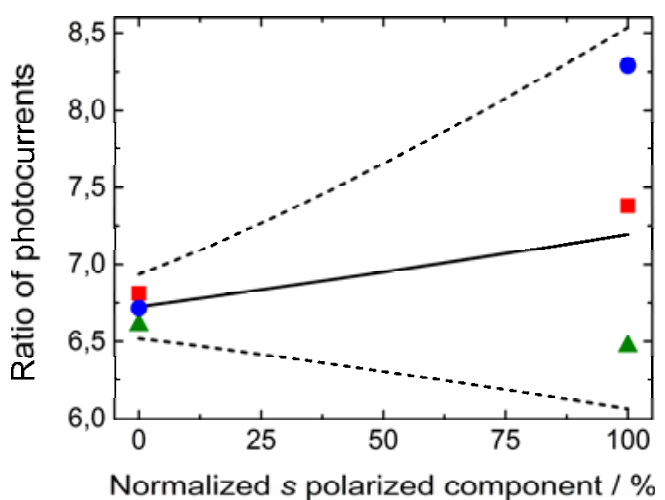

Figure 5. Measured photocurrent ratios of three individual PQEDs (squares, dots and triangles) and calculated value (solid line) at the wavelength of $488 \mathrm{~nm}$ at different combinations of $s$ and $p$ polarized components of the incident laser beam. The dashed lines indicate the standard uncertainties in the calculated value due to detector and photodiode parameters. The uncertainties of the measured values are within the symbol height.

\subsection{Specular reflectance}

Specular reflectance measurements were conducted with the PQED entrance window inclined at Brewster's angle. The uncertainty components due to the window transmittance, reflectance and absorption at $1^{\circ}$ horizontal tilt angle of the PQED are insignificant in reflectance measurements [18]. Measured specular reflectances of nine PQEDs are shown in Figure 6. The values are in agreement with the calculated value of $27.5 \mathrm{ppm}$ with the standard uncertainty of $4.6 \mathrm{ppm}$ [18]. A detailed description of the measurement setup and the uncertainty budget of the measured values are given in [24].

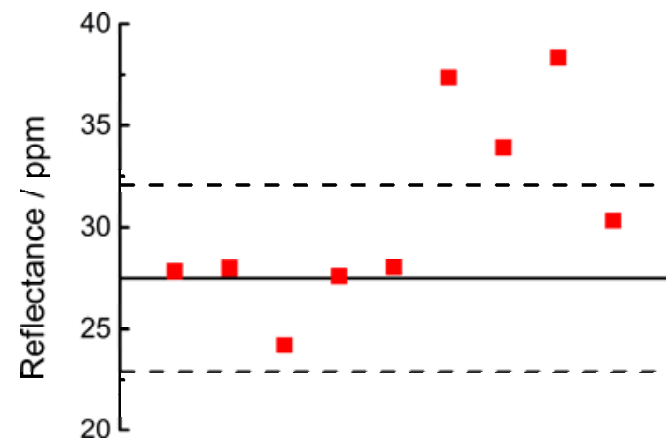

Figure 6. Measured PQED reflectances of $p$ polarized light (squares) and the calculated value (solid line) at the wavelength of $488 \mathrm{~nm}$. The dashed lines indicate the standard uncertainty in the calculated value due to uncertainties in the detector and photodiode parameters [18]. The uncertainties of the measured values are within the symbol height.

\subsection{Relative and absolute IQD}

The internal quantum deficiency (IQD) of three PQEDs was determined. The PQEDs were measured for spectral responsivity against a conventional three element reflection trap detector, traceable to a cryogenic radiometer, by using the method of substitution. The reference trap detector and the PQED were installed on a computer-driven translator and they measured the optical power of the laser beam in the same position. The effect of reflection was then subtracted from the obtained spectral responsivity values. The detailed uncertainty budget of the comparison measurements is given in [24].

The measured IQD values deviate from the average by $-6 \mathrm{ppm}, 4 \mathrm{ppm}$ and $2 \mathrm{ppm}$. The standard uncertainty of the results is $39 \mathrm{ppm}$. These correspond to absolute IQD values of $(-136 \pm 340)$ ppm, $(-126 \pm 340) \mathrm{ppm}$ and $(-128 \pm 340)$ ppm, respectively. The standard uncertainty of the absolute IQD is dominated by the absolute responsivity of the reference trap detector.

\section{Conclusions}

A new compact size room temperature PQED was designed, consisting of two custom designed induced junction photodiodes mounted in a wedged light-trapping configuration. A window at Brewster angle is used in front of the detector to enable high transmission of $p$ polarized light. For improved uncertainty, the window can be removed, in which case a nitrogen flow system is utilized.

Use of $p$ polarized light assures low reflectance losses at the Brewster window and at the photodiodes, whereas $s$ polarized incident light can be used for detector diagnostics. The individual photocurrents of the two photodiodes in the PQED can then provide information on the detector and photodiode alignment. At some wavelengths the reflectance loss of the PQED can be determined from the photocurrent ratio when the incident light has an uncontrolled state of polarization [25].

Measurements of individual detectors at the wavelength of $488 \mathrm{~nm}$ indicate that the spatial responsivity is uniform within at least $50 \mathrm{ppm}$ in the central area of $4 \mathrm{~mm}$ in diameter. The spatial nonuniformity is thus an order of magnitude lower than that of single photodiodes. Single photodiodes show variations of reflectance and responsivity due to nonuniform oxide thickness which seems to be completely eliminated in the PQED, because of the light-trapping structure of the detector.

The reflectance and the IQD of the PQED are congruent within $14 \mathrm{ppm}$ and $10 \mathrm{ppm}$, respectively, and agree with the predicted values. This excellent consistency provides data to support the use of the room temperature PQED as a primary standard of optical power in the visible wavelength range.

\section{Acknowledgements}

The authors acknowledge support by the Academy of Finland grant no 126773. The research leading to these results has received funding from the European Community's Seventh Framework Programme, ERANET Plus, under Grant Agreement No. 217257 and European Metrology Research Programme (EMRP) project SIB57 "New Primary Standards and Traceability for Radiometry". The EMRP is jointly funded by the EMRP participating countries within EURAMET and the European Union. 


\section{References}

1. J. E. Martin, N. P. Fox, P. J. Key, Metrologia 21, 147 (1985)

2. T. Varpula, H. Seppä, J-M. Saari, IEEE Trans. Instrum. Meas. 38, 558 (1989)

3. N. P. Fox, P. R. Haycocks, J.E, Martin, I. Ul-Haq, Metrologia 32, 581 (1995)

4. L. Werner, J. Hartmann, Sens. Actuators A Phys. 156, 185 (2009)

5. J. Geist, App. Opt. 18, 760 (1979)

6. E. F. Zalewski, J. Geist, Appl. Opt. 19, 1214 (1980)

7. J. Geist, E. F. Zalewski, A. R. Schaefer, Appl. Opt. 22, 3795 (1980)

8. J. Geist, E. Liang, A. R. Schaeffer, J. Appl. Phys. 52, 4879 (1981)

9. E. F. Zalewski, C. R. Duda, Appl. Opt. 22, 2867 (1983)

10. T. Hansen, Phys. Scr. 18, 471 (1978)

11. J. Geist, G. Brida, M. L. Rastello, Metrologia 40, S132 (2003)

12. J. Gran, T. Kübarsepp, M. Sildoja, F. Manoocheri, E. Ikonen, I. Müller, Metrologia 49, S130 (2012)

13. M. Sildoja, F. Manoocheri, E. Ikonen, Metrologia 45, 11 (2008)

14. N. P. Fox, Metrologia 28, 197 (1991)

15. J. M. Palmer, Metrologia 30, 327 (1993)

16. J. H. Lehman, Appl. Opt. 36, 9117 (1997)

17. M. Sildoja, F. Manoocheri, E. Ikonen, Metrologia 46, S151 (2009)

18. M. Sildoja, F. Manoocheri, M. Merimaa, E. Ikonen, I. Müller, L. Werner, J. Gran, T. Kübarsepp, M. Smîd, M. L. Rastello, Metrologia 50, 385 (2013)

19. I. Müller, U. Johanssen, U. Linke, L. SocaciuSiebert, M. Smîd, G. Porrovecchio, M. Sildoja, F. Manoocheri, E. Ikonen, J. Gran, T. Kübarsepp, G. Brida, L. Werner, Metrologia 50, 395 (2013)

20. L. Werner, J. Fischer, U. Johannsen J. Hartmann, Metrologia 37, 279 (2000)

21. J. Gran, A. S. Sudbø, Metrologia 41, 204 (2004)

22. Ö. Bazkir, F. Samadov, Opt. Lasers Eng. 43, 131 (2005)

23. M. White, L. Lolli, G. Brida, J. Gran, M. Rajteri, J. Appl. Phys. 113, 243509 (2013)

24. T. Dönsberg, M. Sildoja, F. Manoocheri, M. Merimaa, L. Petroff, E. Ikonen, Metrologia 51, 197 (2014)

25. M. Sildoja, T. Dönsberg, H. Mäntynen, M. Merimaa, F. Manoocheri, E. Ikonen, Meas. Sci. Technol. 25, 015203 (2014) 